\title{
Editorial
}

\section{Computational Methods for Engineering Science}

\author{
K. M. Liew, ${ }^{1}$ L. W. Zhang, ${ }^{2}$ J. N. Reddy, ${ }^{3}$ and Shaofan $\mathrm{Li}^{4}$ \\ ${ }^{1}$ Department of Architecture and Civil Engineering, City University of Hong Kong, Kowloon, Hong Kong \\ ${ }^{2}$ College of Information Technology, Shanghai Ocean University, Shanghai 201306, China \\ ${ }^{3}$ Department of Mechanical Engineering, Texas A\&M University, TX, USA \\ ${ }^{4}$ Department of Civil and Environmental Engineering, University of California, Berkeley, CA, USA
}

Correspondence should be addressed to K. M. Liew; kmliew@cityu.edu.hk

Received 24 March 2015; Accepted 24 March 2015

Copyright (C) 2015 K. M. Liew et al. This is an open access article distributed under the Creative Commons Attribution License, which permits unrestricted use, distribution, and reproduction in any medium, provided the original work is properly cited.

This special issue attempts to cover the recent advances on various aspects of theories, analyses, and applications of computational methods in engineering science, reflecting the state of the art in computational methods and their frameworks, applications, networking technologies, and new and advanced engineering applications in emerging technologies such as the bioscience and biotechnology, nanoscience and nanotechnology, numerical modeling, simulation and analysis, and material sciences.

The topics covered by the articles including in this special issue encompass computational mechanics, ocean and offshore engineering, computational fluid dynamics, computational mathematics and statistics, computational physics, computational material sciences, multiscale modeling, disaster simulation and analysis, element-free/meshless/mesh-free methods and dimension-reduction methods, geometric and material nonlinear analyses, damage, fracture and fatigue, contact mechanics and friction, smart structures and health monitoring, structural optimization, nanomechanics, biomechanics, inverse and coupling problems, and reliability theory and application.

We hope that this special issue will be cited for recent advances in these research areas.

K. M. Liew L. W. Zhang 


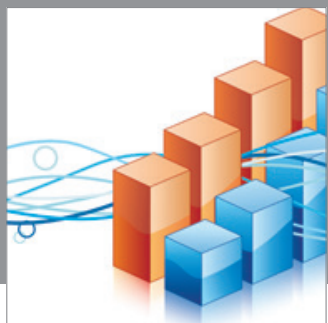

Advances in

Operations Research

mansans

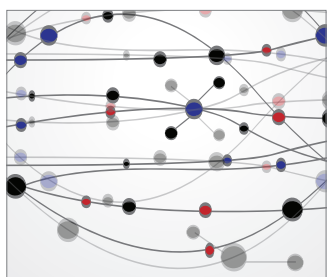

The Scientific World Journal
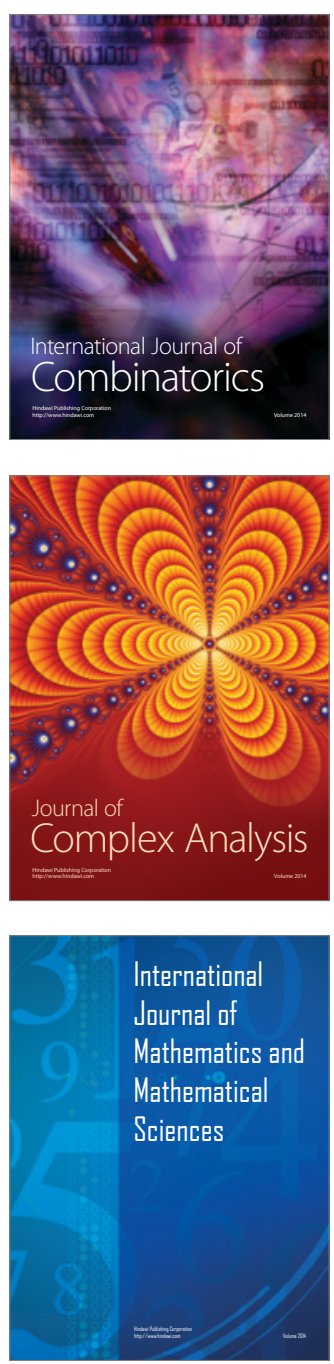
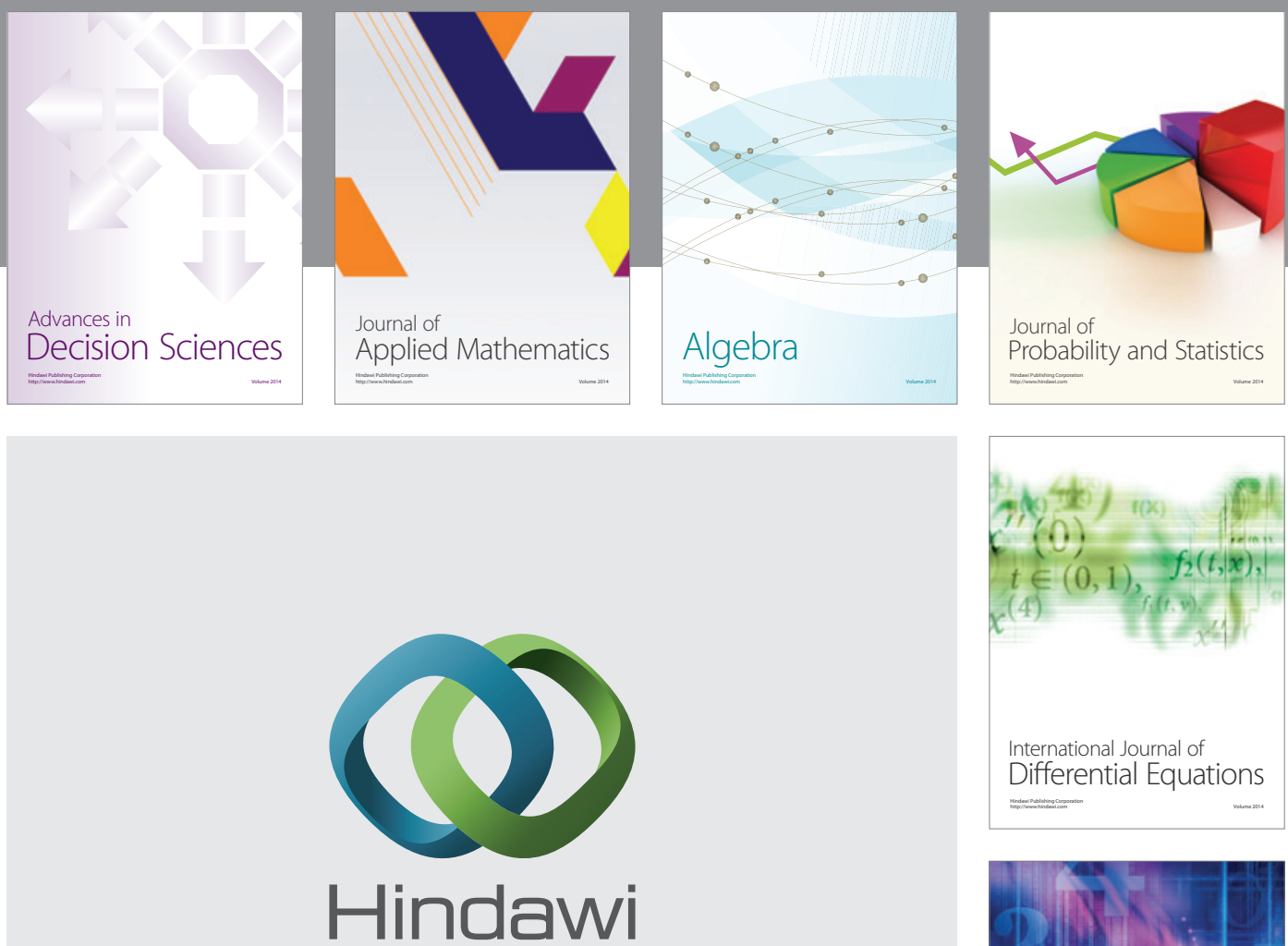

Submit your manuscripts at http://www.hindawi.com
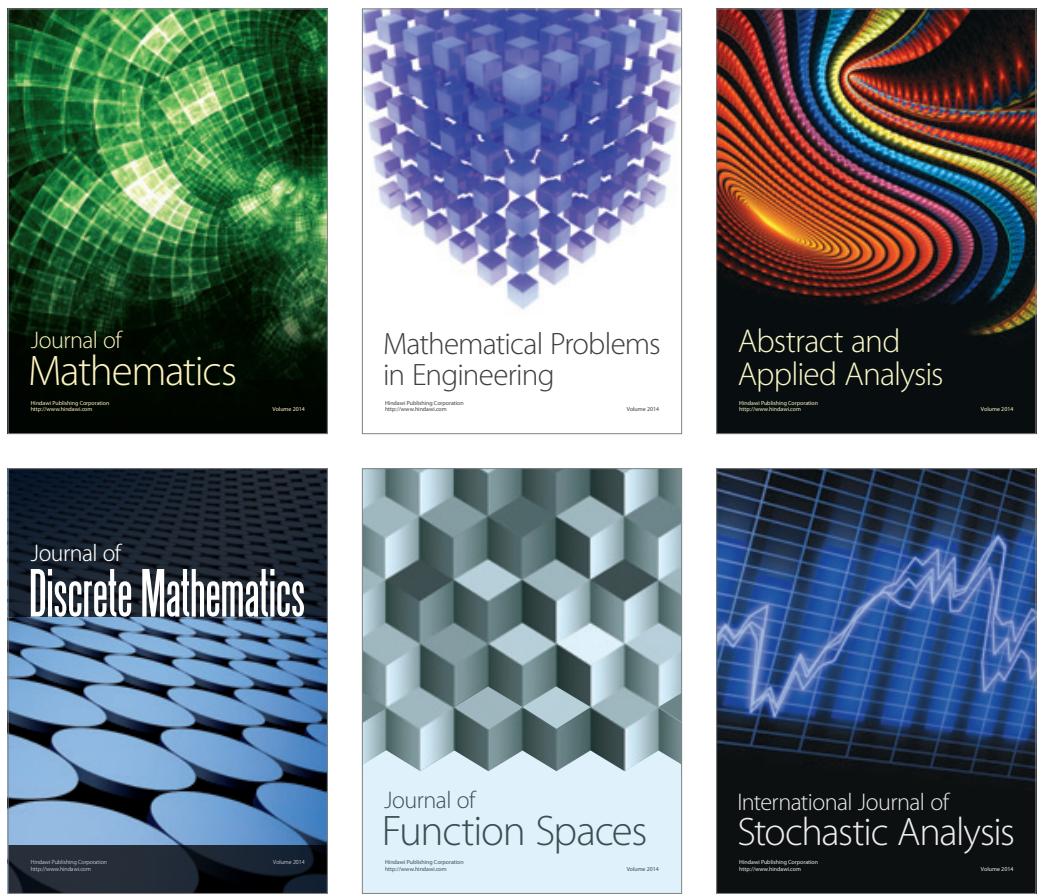

Journal of

Function Spaces

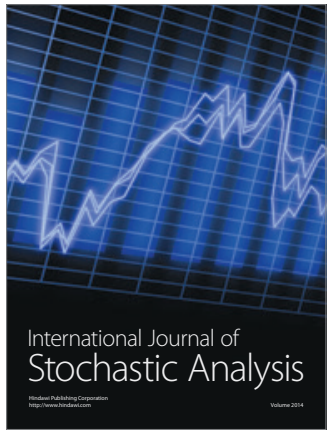

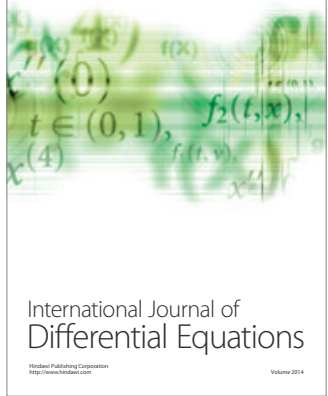
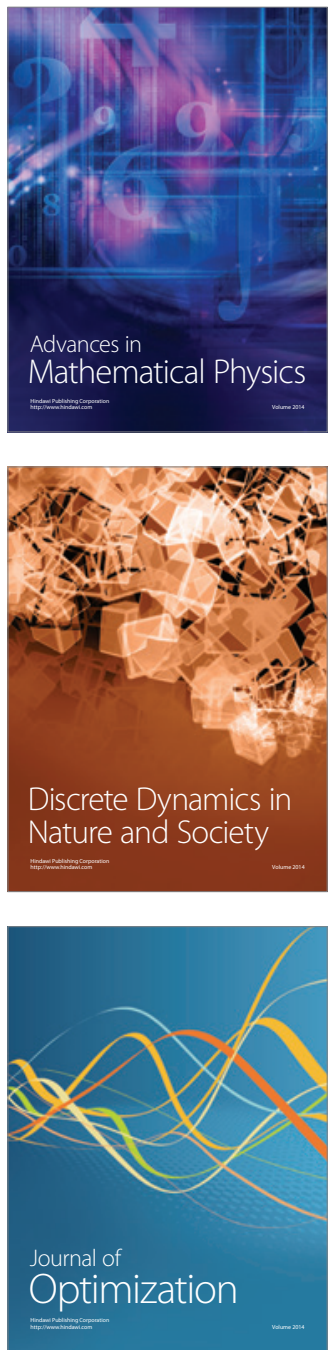